\title{
Editorial: Special Issue on Intelligent RFID Network and Innovative Applications
}

\author{
Sy-Yen Kuo • Chang-Fa Yang • \\ Shuo-Yan Chou · Jiann-Liang Chen
}

Published online: 2 December 2010

C) Springer Science+Business Media, LLC. 2010

Recent developments in the field of radio frequency technologies have led to numerous challenges in providing comprehensive performance for the next generation wireless networks. Meanwhile RFID as the one of important core technology in toward this future approach, have been implemented extensively for different kind applications. Those applications direct to the obviousness that issues in electromagnetic, signal collusion, privacy and security have decreased RFID role in industry implementation. In designing RFID devices more challenges and difficulties are become significant due to the low-cost and low-source factors. This has encouraged researchers to think hard in developing more secure and accurate RFID tags.

Therefore this edition present the papers that addressing the solution diverse issues in RFID system such as: tag antenna and propagation, communication protocol, security and privacy, system tools, RF-based localization, RFID system architecture, deployment issue, and also applications.

Chi-Yi Lin and Wen-Tsung Cheng from Tamkang University, Szu-Chi Wang from National I-Lan University implemented hybrid system integrating GPS and RFID technologies to address three important factors in providing detail and reliable end to end logistics managements. Their implementation focuses in multiple heterogeneous location system in approach to extend functionality in information delivering method.

\section{S.-Y. Kuo}

Department of Electrical Engineering, National Taiwan University, Taipei, Taiwan, ROC

e-mail: sykuo@cc.ee.ntu.edu.tw

C.-F. Yang · J.-L. Chen $(\bowtie)$

Department of Electrical Engineering, National Taiwan University of Science and Technology, Taipei, Taiwan, ROC

e-mail: Lchen@mail.ntust.edu.tw

C.-F. Yang

e-mail: cyang@mail.ntust.edu.tw

\section{S.-Y. Chou}

Department of Industrial Management, National Taiwan University of Science and Technology,

Taipei, Taiwan, ROC

e-mail: sychou@mail.ntust.edu.tw 
JingHuey Khor, Widad Ismail, Mohammed I. Younis, M.K. Sulaiman, and Mohammad Ghulam Rahman from University Science Malaysia investigated security and privacy issues in low cost RFID system to the three most intriguing threat types, and by implementing fingerprint system they convince that prevention and detection strategy using lightweight cryptographic algorithm of EPC C-1 Gen-2 are effective to improve RFID security level.

Joan Meliá-Seguí from Universitat Oberta de Catalunya, Joaquin Garcia-Alfaro from Institut Telecom Bretagne and Jordi Herrera-Joancomartí from, Universitat Autònoma de Barcelona investigated the security weakness of EPC Gen2 tags by perform a practical implementation attack using weak PRNG. They enact that in order to determine certain PRNG configuration for comprehensive output sequence, small amount of pseudorandom values is feasible to be eavesdropped by standard EPC command.

SSM and LRMAP protocol is examined by Imran Erguler and Emin Anarım from Boğaziç University, they conclude at certain cases and arrangements of generic attack, these protocols are failed to accomplish RFID tradeoff on scalability toward privacy and security issue.

Antti Ropponen, Henry Rimminen, and Raimo Sepponen from Aalto University did a useful contribution for health care environment system for indoor localization and identification by improving range of detection range and antenna laminate. A setup experiment by quad antennas and $125 \mathrm{kHz}$ radio tag signal confirmed the robustness of their proposed system.

Chu-Sing Yang from National Cheng Kung University, Yu-Chun Lu, Ming-yi Liao, Chaoxing Chen from National Sun Yat-sen University design the HEMS (Household Effects Management System), a system constructed by RFID and OSGi implementation. By querying certain objects, HEMS provides a remainders system for people travel away from the house.

Wei Chen, Wenyi Che, Na Yan, Xi Tan and Hao Min from Fudan University contribute their work on designing low power Truly Random Generator (TRNG) for EPC Gen-2 for RFID with considering randomness and chip area tradeoffs issue.

Yi-Wei Ma from National Cheng Kung University, Chin-Feng Lai Chia Nan University of Pharmacy \& Science Tainan, Jenq-Muh Hsu, Nong-Kun Chen from National Chiayi University, and Yueh-Min Huang National Dong-Hwa University address the accuracy as main goal in improving telematics location aware application design based on RFID tag implementation. This work achieves the improvement by adopt and extending LANDMARC algorithm.

Der-Jiunn Deng and Hsuan-Wei Tsao from National Changhua University of Education identify two weaknesses of DFSA algorithm: low throughput and bad power consumption due to its poor capability in managing the frame size. By conducting analysis and simulation to address the solution on frame size adjustment they are able to show better performance.

Hsin-Chin Liu and Che-Rong Kuo from National Taiwan University of Science and Technology perform an improvement in expanding passive RFID reader coverage, base their work on the continuous waves in multiple frequency bands a method in analyzing multi carrier.

Yuan-Chih Lin, Tzu-Chen Hung, and Chii-Ruey Lin from National Taipei University of Technology present a novel antenna design for IEEE 802.15 with coupling feeding mechanism, this antenna can be easily toned on $2.4 \mathrm{GHz}$ and $50 \mathrm{ohm} \mathrm{RF}$ input specification, moreover their work is able achieve half wavelength of current loop which enable the antenna implementation on very limited space.

Yuan-Ping, Luh and Yin-Chang, Liu from National Taipei University of Technology present an optimal Q parameter to become a control module in UHF RFID interrogator applied in EPC gen 2 standard of RFID tag, by proper Q value adjustment very high reading rate can be achieved by their design.

These great works have given a massive contribution for RFID research area. Therefore editor would like to thank all paper contributors for their brilliant idea, their hard work in preparing the paper, and for their faithfulness in follow the reviewing process. We also thank 
the reviewers for their work to analyze submitted paper and improve accepted paper to be more proper to present its key technologies. By their work we are able to keep journal's track in presenting high quality research result; therefore we would like to express our apologies for the authors where in this occasion cannot get the opportunity to present their research in this journal.

\section{Author Biographies}
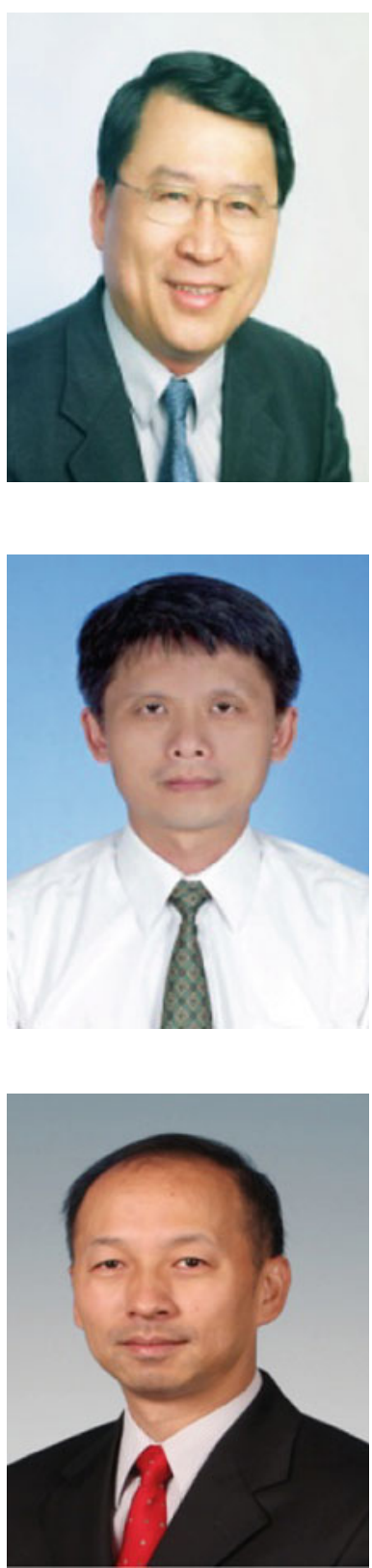

Sy-Yen Kuo is a Distinguished Professor at the Department of Electrical Engineering, National Taiwan University, Taipei, Taiwan and was the Chairman from 2001 to 2004. He was a Dean of the College of Electrical and Computer Engineering, National Taiwan University of Science and Technology from 2006 to 2009. He received his Ph.D. (1987) in Computer Science from the University of Illinois at UrbanaChampaign. He is an IEEE Fellow. He has published more than 300 papers in journals and conferences, and also holds several patents. His current research interests include dependable systems, mobile computing and quantum computing.

Chang-Fa Yang received his B.S. Degree from National Taiwan University in 1983 and the Ph.D. degree from the Ohio State University, Columbus in 1992, in Electrical Engineering. From 1986 to 1992, he was a graduate research associate with the ElectroScience Laboratory. In February 1992, he joined the faculty in the Department of Electrical Engineering, National Taiwan University of Science and Technology (NTUST). He has been a full Professor since 1999 and a Director of the NTUST Wireless Communication and Electromagnetic Compatibility Research Center since 2005. His research interests include wave propagation, antenna design, and electromagnetic compatibility.

Shuo-Yan Chou is a Professor of Industrial Management and the Executive Secretary of the RFID Education and Research Center at the National Taiwan University of Science and Technology. His research interests include demand modeling, supply chain management, intelligent system modeling and application, and geometric algorithms. He is the Editor-in-Chief of the Journal of Chinese Institute of Industrial Engineers. He was a visiting scholar at Hong Kong University of Science and Technology, University of Washington (Seattle) and an Adjunct Professor at Beijing Jiaotong University. He received his Ph.D. in Industrial and Operations Engineering from the University of Michigan in 1992. 


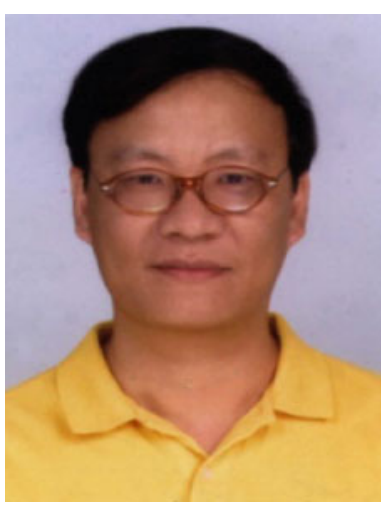

Jiann-Liang Chen received his Ph.D. Degree in Electrical Engineering from the National Taiwan University, Taipei, Taiwan in 1989. Since August 1997, he has been with the Department of Computer Science and Information Engineering of National Dong Hwa University, where he is a Professor and Vice Dean of the Science and Engineering College. He is joining the Department of Electrical Engineering, National Taiwan University of Science and Technology as a full Professor now. His current research interests are in mobile computing, digital home network, telematics applications, and RFID middleware design. 